\title{
COMPARISON OF TRANSCRANIAL DOPPLER ULTRASOUND WITH COMPUTATIONAL FLUID DYNAMICS: RESPONSES TO PHYSIOLOGICAL STIMULI
}

5

6

7

8

9

10

\author{
HARRISON T. CADDY ${ }^{1,2}$ \\ HANNAH J. THOMAS ${ }^{3}$ \\ LACHLAN J. KELSEY ${ }^{1,2}$ \\ KURT J. SMITH ${ }^{3,4}$ \\ BARRY J. DOYLE ${ }^{1,2,5,6^{*}}$ \\ DANIEL J. GREEN ${ }^{3 *}$
}

\footnotetext{
${ }^{1}$ Vascular Engineering Laboratory, Harry Perkins Institute of Medical Research, Queen Elizabeth II Medical Centre, Nedlands, Australia and the UWA Centre for Medical Research, The University of Western Australia, Perth, Australia

${ }^{2}$ School of Engineering, The University of Western Australia, Perth, Australia

${ }^{3}$ School of Human Sciences (Exercise and Sport Sciences), The University of Western Australia, Perth, Australia

${ }^{4}$ Integrative Physiology Laboratory, Department of Kinesiology and Nutrition, University of Illinois, Chicago, Illinois

${ }^{5}$ Australian Research Council Centre for Personalised Therapeutics Technologies, Melbourne, Australia

${ }^{6}$ British Heart Foundation Centre for Cardiovascular Science, The University of Edinburgh, Edinburgh, United Kingdom

* Joint senior authors
}

SHORT TITLE: Comparison of TCD and CFD in response to stimuli

AUTHOR FOR CORRESPONDENCE:

Associate Professor Barry J Doyle

barry.doyle@uwa.edu.au

The University of Western Australia (M519),

35 Stirling Highway, 6009 Perth, Australia 
Caddy et al. Comparison of TCD and CFD in response to stimuli

\section{ABSTRACT}

Few studies have compared transcranial Doppler (TCD) ultrasound with independent techniques such as computational fluid dynamics (CFD) simulations, particularly in response to stimuli. We compared TCD cerebral blood flow velocity in healthy participants with subjectspecific CFD simulations to determine differences in techniques. Twelve participants underwent head and neck imaging with 3 Tesla magnetic resonance angiography. Velocity waveforms in the middle cerebral artery (MCA) were measured with TCD while velocity and diameter in the neck arteries were measured with duplex ultrasound at rest, hypercapnia and exercise. Subject-specific CFD simulations were developed for each condition, with velocity waveforms extracted in the same region as TCD. We found that absolute TCD velocities were significantly higher than CFD data, and non-significantly correlated across all conditions ( $r$ range $0.030-0.377$, all $P>0.05)$. However, relative changes from rest to hypercapnia and exercise generally exhibited significant positive correlations ( $r$ range $0.448-0.770$ ), with the strongest correlation being average velocity change from rest to exercise $(r=0.770, P<0.01)$. We have found that although absolute MCA velocity measurements from different sources vary, relative velocity changes yield stronger correlations regardless of source. Our findings indicate relative responses to physiological stimuli, along with absolute data, should be considered for analyzing cerebral blood flow velocity.

\section{Key Terms}

Cerebrovasculature; computational fluid dynamics; transcranial Doppler ultrasound; comparison; stimuli 
Caddy et al. Comparison of TCD and CFD in response to stimuli

\section{INTRODUCTION}

58 Transcranial Doppler (TCD) is an ultrasound technique which is commonly used for measuring

59 blood flow velocities within the brain. It is typically used to insonate the middle (MCA), posterior (PCA) or anterior cerebral arteries via either the transtemporal, transorbital or suboccipital windows. A conventional TCD system consists of a low frequency $2 \mathrm{MHz}$ probe and is either held in location by an operator, or secured using a head brace ${ }^{42}$. TCD is beneficial for measuring cerebral blood flow velocity because it is non-invasive, reproducible, can be quickly performed in real-time, is portable and possesses high temporal resolution ${ }^{42}$. Despite these benefits, results are known to be operator dependent ${ }^{25}$, as well as limited by fixed transorbital windows and the probe frequency required to transit the bony skull. Furthermore, TCD can only measure velocity, as B-mode images of diameter cannot be simultaneously measured due to resolution constraints ${ }^{42}$.

TCD has been used to measure blood flow velocity and derived metrics of arterial pulsatility and resistance in patients with cerebrovascular diseases $4,13,18$, as well as being used in estimating cerebral blood flow (CBF) to different regions of the brain ${ }^{24}$. It has also been used to assess prognosis and diagnosis of life-threating conditions such as sickle cell anemia, stenosis, hemorrhage and ischemia across a spectrum of patient ages ${ }^{25,29}$. TCD is also used extensively in healthy populations to better understand cerebrovascular responses to physiological stimuli including hypercapnia, hypocapnia, exercise, vessel occlusion, shearmediated endothelial responses and neurovascular coupling 5, 11, 15, 28, 41 . In addition, measurement of blood flow velocity using TCD has been used to infer volumetric cerebral blood flow - however the absence of diameter measurement is a limitation in this regard. 
81 velocities during the vasodilatory stimuli of hypercapnia ${ }^{43}$ and exercise ${ }^{39}, 40$, suggest intracranial diameter changes may result in underestimation of CBF when estimated from

83 TCD ultrasound. There is also some evidence which suggests that cerebral artery diameter may vary within the cardiac cycle ${ }^{1,7}$ and that these arteries are also known to vasoconstrict in response to changes in arterial blood pressure in the context of cerebral autoregulation ${ }^{3,}$ 11. Consequently, using TCD velocities as a measure proportional to cerebral blood flow remains controversial in the absence of a B-mode image of diameter change ${ }^{1}$. In some instances, this limitation has been addressed by using ICA or vertebral arteries (VA) as surrogates for intra-cranial vessels, which are contiguous with arteries such as the MCA and PCA, and can be imaged using real time duplex ultrasound.

Few studies have compared measurements of blood flow velocity obtained using TCD with alternative and independently derived techniques. One such alternative method for measuring blood flow within the brain is through magnetic resonance imaging (MRI), such as magnetic resonance angiography (MRA). Despite limitations in temporal resolution, this approach possesses high spatial resolution and is able to capture the complex three dimensional (3D) nature of cerebral arteries ${ }^{1}$. Computational fluid dynamics (CFD) simulations provide a method for combining the strengths of both MRA and duplex ultrasound to calculate fluctuations of blood flow velocity within the cerebral vasculature with high spatial and temporal resolution. Although CFD has been used in conjunction with TCD methods for independent comparison ${ }^{14,27,30,35}$ under static conditions, few studies have investigated velocities measured using TCD with independent methods, such as CFD, in response to common physiological stimuli ${ }^{11}$. 


\section{Caddy et al. Comparison of TCD and CFD in response to stimuli}

105

106

107

108

109

110

111

In this study, we tested the change in TCD velocity measurements in response to physiologically relevant stimuli such as hypercapnia and exercise, and compared these data to changes in simulated cerebrovascular velocities calculated using CFD methods. Our CFD approach combined ICA and VA duplex ultrasound measurements to prescribe volumetric flow inlet boundary conditions, and used 3D geometry based on individualized MRA-derived cerebrovascular reconstructions. We hypothesized that TCD- and CFD-derived velocity metrics would be similar and highly correlated at rest and in response to physiological stimuli.

\section{MATERIALS AND METHODS}

\section{Participant Cohort and Medical Imaging}

The experimental procedures used in this study were approved by The University of Western Australia Human Research Ethics Committee. A total of twelve healthy participants ( 6 female, 6 male) were recruited for this study with ages ranging between 19 and 28 years old. Participants were made aware of the experimental procedure and associated risk. Written consent was obtained for each participant prior to commencement of the experimental study. Prior to cerebrovascular stimuli, each participant underwent a 3 Tesla time-of-flight (3T TOF) MRA (Siemens MAGNETOM Skyra) neck and head scan. This scan had a pixel size of $0.31 \mathrm{~mm}$ and a slice thickness of $0.75 \mathrm{~mm}$.

\section{Cerebrovascular Stimuli Procedure}

Participants were exposed to conditions previously described by Thomas et al. ${ }^{41}$, consisting of rest ( 5 minutes recumbent), hypercapnia ( 5 min of $6 \% \mathrm{CO}_{2}$ via Douglas bag recumbent) and submaximal exercise (5 min recumbent cycling at 90 Watts) conditions respectively. Each session was conducted in the morning for all participants, who were instructed to fast (no 


\section{Caddy et al. Comparison of TCD and CFD in response to stimuli}

129

130

131

132

133

134

135

136

137

138

140

141

142

143

144

145

146

147

food, tea or coffee) and abstain from consumption of alcohol or performing exercise in the 24 hours prior to the session. After each of the exposure condition durations, we simultaneously measured velocity (Doppler ultrasound) and intraluminal diameter (B-mode ultrasound) in the left and right ICAs and VAs using two identical 10-MHz linear array probes and high-resolution ultrasound machines (Terason 3200, Teratech, Burlington, MA) using standardized search techniques ${ }^{42}$. Continuously during each condition, we measured the peak velocity envelope in the MCA, specifically in the right M1 segment via the middle transtemporal window using a 2-MHz TCD probe (TCD, Spencer Technologies, Seattle, WA) which was held in place with a headpiece (M600 bilateral head frame, Spencer Technologies) as per methods described previously ${ }^{15}$. Throughout all exposure conditions, we continuously measured end-tidal partial pressure of $\mathrm{CO}_{2}$ and $\mathrm{O}_{2}$ using a gas analyzer (Gas Analyzer, ADInstruments, New South Wales, Australia). For analysis and calculation of ICA and VA flow and TCD MCA velocities, we considered data sampled within the final 30 second period of each 5 minute exposure condition. To mitigate cerebral priming, participants underwent a 10 minute washout period, remaining in a recumbent position, between each exposure condition, which was sufficient to return metrics (mean arterial pressure, heart rate, end tidal $\mathrm{CO}_{2}$, average MCA velocity from $\mathrm{TCD}$ ) to baseline levels across all participants.

\section{MRA Reconstruction and Ultrasound Analysis}

\section{MRA 3D Reconstruction}

To create the 3D fluid domain for the CFD simulations, we imported the DICOM images from the MRA scan into in-house image reconstruction software. We used region-growing techniques to select similar intensity labeled pixels greater than an intensity value of 200 to 
Caddy et al. Comparison of TCD and CFD in response to stimuli

152

153

154

155

156

157

158

159

160

161

162

163

164

165

166

167

outline the fluid contained within the cerebrovascular geometry. The software uses an inbuilt marching cubes algorithm to create a 3D isosurface. This isosurface was globally smoothed to within $5 \%$ of the starting volume, which was then imported into STAR-CCM+ $(v 12$, Siemens, Munich, Germany) to perform surface repair, remove reconstruction artifacts and perform local smoothing. Outlets were truncated perpendicular to the vessel centerline at least two bifurcations downstream from the Circle of Willis (CoW).

\section{Duplex and TCD Ultrasound}

The duplex ultrasound measurements of diameter and velocity at the ICAs and VAs over three cardiac cycles ${ }^{20}$ were converted to time varying volume flow rate and waveform averaged and processed as described previously ${ }^{41}$. This resulted in spline fitted and peak aligned data. Similarly, TCD maximal velocity waveform data from three cardiac cycles within the right M1 segment (Figure 1) were combined into an average waveform using the same process for each participant.

\section{Computational Fluid Dynamics}

\section{Computational Mesh}

The simulations were developed in the commercial CFD package STAR-CCM+. We used a combination of a polyhedral element mesh for the core of the fluid domain and 20 prism layer elements in the near wall boundary to sufficiently capture the velocity gradients and ensure accurate calculation of wall shear stress. In addition, we prescribed extrusions at the fluid boundaries equal to 11 times the boundary diameter to ensure adequate development of parabolic flow upstream and downstream of the fluid domain ${ }^{6}$. Mesh core density was set 
proportional to local vessel diameter and the mesh settings were prescribed as per previously published work ${ }^{41}$. These settings had been optimized to ensure mesh independence using

177 the grid convergence index ${ }^{31}$ and used the subject with the greatest inlet velocity measurements for this optimization. Optimal mesh size was deemed sufficient when the grid convergence index for wall shear stress within the CoW was found to fall below $3 \%{ }^{41}$. Final mesh sizes ranged from 9.1 to 16.7 million cells per geometry.

\section{Boundary Conditions}

Boundary conditions followed methods as described previously ${ }^{41}$. Briefly, the three cardiac cycle duplex ultrasound measurements of velocity and diameter obtained at the ICAs and VAs were converted from the calculated volumetric flowrates to mass flow waveforms, assuming a fluid density of $1050 \mathrm{~kg} \mathrm{~m}^{-3} 22$. The measured velocity waveforms for the rest, hypercapnia and submaximal exercise conditions were all processed using this method and prescribed at the corresponding extruded inlet using a plug flow condition, which developed into a parabolic profile throughout the extruded inlet region before entering the subject specific cerebrovascular geometry. Outlet boundary conditions were implemented using the same WALNUT code described previously ${ }^{41}$, which initially splits blood flow exiting the fluid domain into seven regions (left and right posterior, left and right middle, anterior, cerebellum and ophthalmic arteries) and accounts for the presence or absence of communicating arteries in the CoW, with different flow distributions to these regions based on the average volumetric

195 flow measured from the ICAs and VAs. Within these regions, flow was then split using an adaptation of the Murray's law formulation ${ }^{10}$ as defined in equation 1 , using an exponent of $n=2.33^{41}$. 


$$
Q_{o u t, i}=Q_{\text {in }, \text { region }} \times \frac{d_{i}^{n}}{\sum_{j=1}^{N} d_{j}^{n}}
$$

198

199

200

201

202

203

204

205

206

207

208

209

210

211

212

213

214

215

216

217

218

219

Where $Q_{\text {out }, \text { is }}$ is the flow out of outlet $i, Q_{\text {in, region }}$ is the WALNUT calculated flow into the region of the cerebrovasculature which is shared by outlet $i$ as described previously ${ }^{41}, d$ is outlet diameter and $n$ is the flow split exponent.

\section{Physical Assumptions}

Blood was assumed incompressible with a density of $1050 \mathrm{~kg} \mathrm{~m}^{-3} 22$. The non-Newtonian nature of blood viscosity was modelled using the Carreau-Yasuda viscosity model using parameters appropriate for blood flow within the CoW ${ }^{19}$. We assumed the arterial walls were rigid, with a no-slip boundary condition and a laminar flow regime in line with previous cerebrovascular simulations ${ }^{2,19,36,41}$.

\section{Simulation Execution}

Simulations ran for three consecutive cardiac cycles to ensure flow stabilization, with results extracted from the fourth cardiac cycle. The implicit unsteady segregated flow solver was employed, which uses the Semi-Implicit Method for Pressure Linked Equations (SIMPLE) for coupling the velocity and pressure components of the Navier-Stokes equations. We used second-order temporal discretization with an automated time-step control which permitted the time-step to vary between 0.001 and $0.005 \mathrm{~s}$ depending on the Courant number. New time-steps were triggered if absolute continuity and momentum residuals fell below a value of $10^{-9}$, or if the number of inner iterations reached 50 . Our simulations were executed using the STAR-CCM+ finite-volume method on Magnus, a Cray XC40 supercomputer (Pawsey Supercomputing Centre, Perth, Australia) housing a total of 1488 compute nodes each 
containing 24 cores per node. We ran each simulation utilizing 25 nodes over a collective of 500 cores. The rest, hypercapnia and exercise simulations required an average of 3000,2800 and 2000 core hours respectively to run, which equated to total simulation run times ranging from approximately 3 to $7 \mathrm{~h}$.

\section{Data Collection, Analysis and Statistics}

226 Simulations were allowed to stabilize over three cardiac cycles, after which we extracted maximal velocity waveforms from each simulation over the fourth cardiac cycle using the average of the maximum value from three consecutively spaced constrained planes located within the right M1 segment (Figure 1 ) to represent the region insonated using TCD. The velocity waveforms extracted from the simulation and from the TCD ultrasound envelope for each participant and exposure condition were analyzed for their characteristics using a custom MATLAB script (R2016, Mathworks, Natick, MA). We extracted the systolic, average and end-diastolic maximal velocities from these waveforms. We used paired t-tests for comparison of distributions and Pearson's correlation and Bland-Altman plots to investigate

235 the correlations between absolute and relative change in CFD and TCD data for each participant in response to different stimuli. Normality was tested using the Shapiro-Wilk test.

237 Where applicable, data is presented as group mean \pm standard deviation. Statistical significance was assumed for $p$-values where $P<0.05$. 


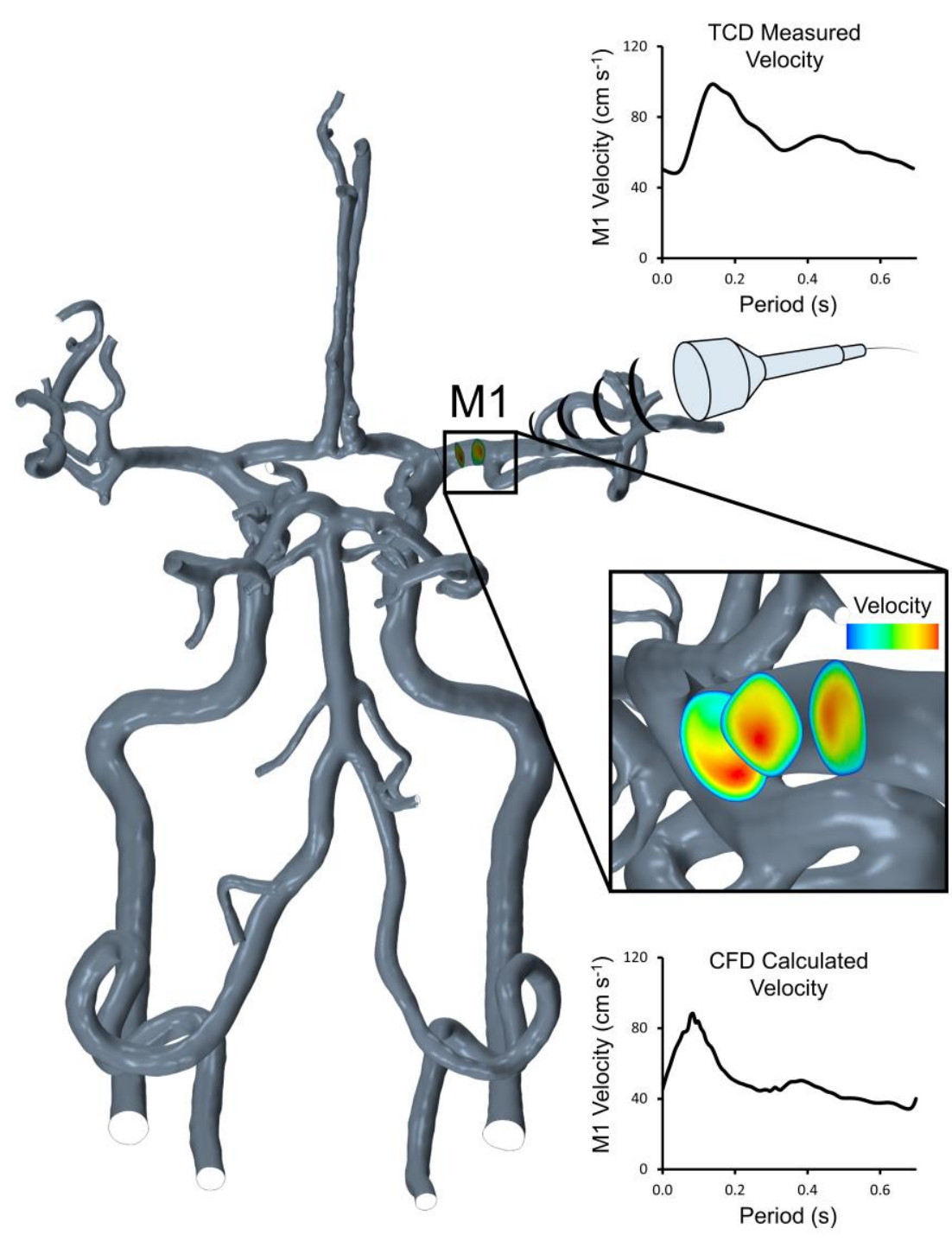

240 Figure 1. Example TCD probe (top) and consecutively spaced CFD constrained planes (bottom)

241 demonstrating sampling of data within the right M1 segment. Within the CFD simulations,

242 maximal velocity was calculated and extracted at each of these planes and averaged into a

243 mean maximal value representative of the peak velocity envelope from TCD insonation of the

244 right M1 segment in each participant. Example velocity waveforms from TCD and CFD sources

245 from an individual are provided. Characteristics from these velocity waveforms were then

247 (rest, hypercapnia and exercise). 


\section{Caddy et al. Comparison of TCD and CFD in response to stimuli}

\section{RESULTS}

\section{Participant Cardiorespiratory Responses and MRA Reconstructions}

251 Participants had an average age, $\mathrm{BMI}$ and $\mathrm{VO}_{2}$ max score of $22.9 \pm 3.4$ years, $21.6 \pm 2.9 \mathrm{~kg} \mathrm{~m}^{-2}$ and $44.7 \pm 9.4 \mathrm{~mL} \mathrm{~kg}^{-1} \mathrm{~min}^{-1}$ respectively. Participant cardiorespiratory responses including end tidal $\mathrm{CO}_{2}$, blood pressure, heart rate as well as ultrasound derived average volumetric

Table 1. Participant cardiorespiratory responses under different conditions.

\begin{tabular}{lrrr}
\hline & \multicolumn{1}{c}{$\begin{array}{c}\text { Rest } \\
\text { (Average } \pm \text { SD) }\end{array}$} & $\begin{array}{c}\text { Hypercapnia } \\
\text { (Average } \pm \text { SD) }\end{array}$ & $\begin{array}{c}\text { Exercise } \\
\text { (Average } \pm \text { SD) }\end{array}$ \\
\hline End-tidal CO $(\mathrm{mmHg})$ & $42.2 \pm 2.5$ & $50.7 \pm 1.9$ & $43.8 \pm 4.2$ \\
Mean arterial pressure $(\mathrm{mmHg})$ & $100.0 \pm 8.1$ & $106.0 \pm 11.8$ & $124.8 \pm 15.2$ \\
Heart rate $(\mathrm{bpm})$ & $68.7 \pm 9.5$ & $74.1 \pm 8.1$ & $120.4 \pm 21.3$ \\
Mean blood flow $\left(\mathrm{mL} \mathrm{min}^{-1}\right)$ & & & \\
LICA & $268.9 \pm 63.3$ & $374.6 \pm 79.3$ & $272.8 \pm 67.2$ \\
RICA & $218.8 \pm 74.2$ & $308.6 \pm 79.3$ & $262.9 \pm 50.3$ \\
LVA & $74.2 \pm 26.4$ & $119.4 \pm 30.9$ & $79.7 \pm 27.1$ \\
RVA & $56.2 \pm 18.3$ & $97.0 \pm 32.3$ & $68.4 \pm 34.0$
\end{tabular}

260

261 Data are mean \pm standard deviation. LICA = left internal carotid artery; RICA = right internal 
Caddy et al. Comparison of TCD and CFD in response to stimuli

\section{Absolute Velocity Data Distributions at Single Conditions}

265 In general, measurements of maximal systolic, average and end diastolic velocity were all

266 significantly higher (all $\mathrm{P}<0.05$ ) in the TCD data compared to the CFD simulations (Figure 2 ).

267 The mean of the systolic velocity distribution ranged from 33-73\% higher in the TCD data

268 compared to CFD across the exposure conditions. Greater differences in means were

269 observed for average velocity, with increases ranging from $62-85 \%$ when comparing TCD to

270 CFD data across all exposure conditions. We observed the highest changes in end diastolic

271 velocity, with increases ranging from $85-106 \%$ between TCD and CFD data across all

272 conditions. 
Rest
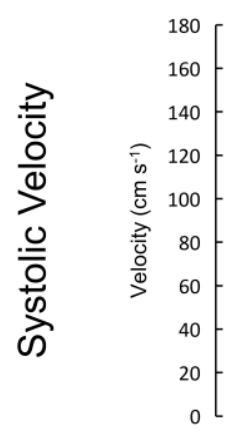

त्ञ

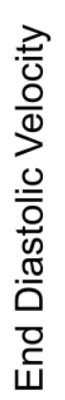

273
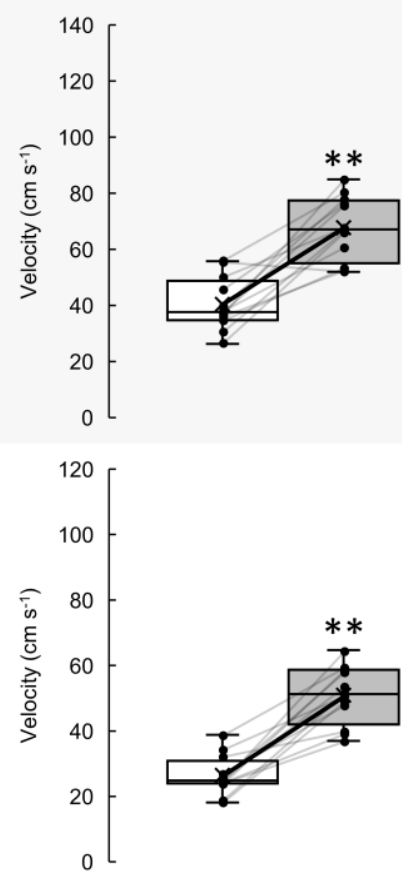

Hypercapnia
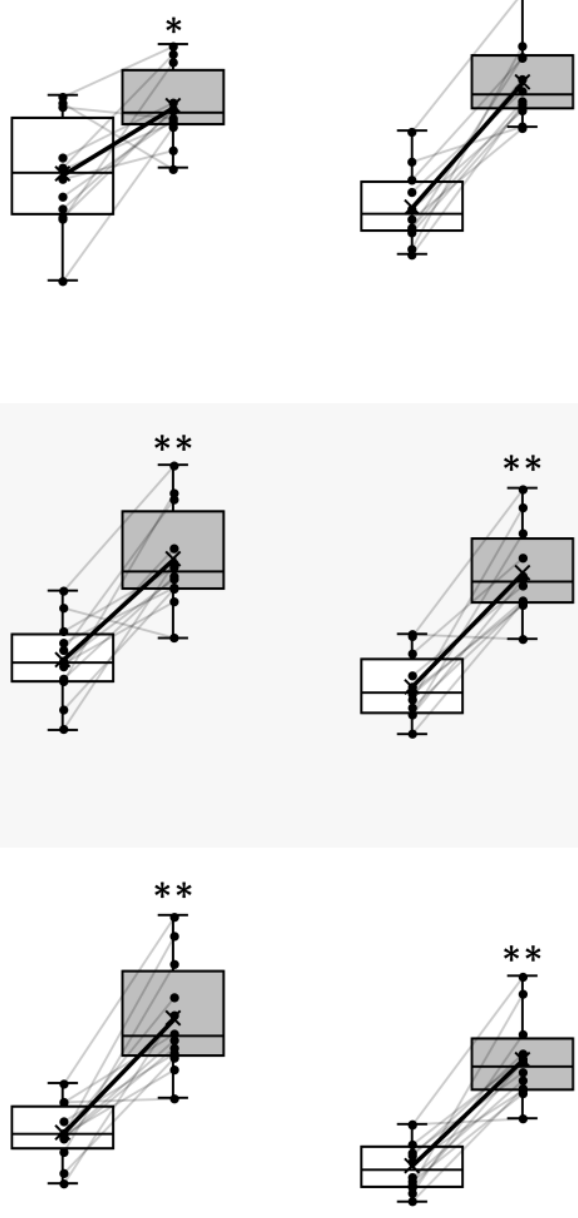

Exercise
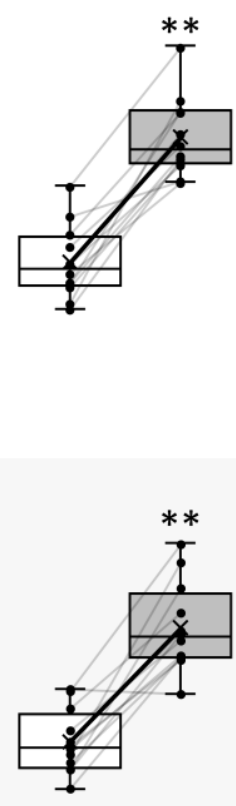

CFD Data

TCD Data
274 collected for each of the stimuli conditions of rest, hypercapnia and exercise. Stars $\left({ }^{*}\right)$ indicate

Figure 2. Box plot distributions of systolic, average and end diastolic maximal velocity extracted from CFD (white; $n=12 ; 6$ male, 6 female) and three cycle averaged TCD (grey; $n=12$; 6 male, 6 female) data in the right M1 segment. Individual differences between CFD and TCD data are presented as black dots with grey connecting lines. The solid black line connecting the cross $(X)$ in each box indicates the changing means of the distributions. These data were the level of significance $\left({ }^{*} \mathrm{P}<0.05 ;{ }^{*} \mathrm{P}<0.001\right)$ using t-tests between CFD and TCD data. 
Caddy et al. Comparison of TCD and CFD in response to stimuli

282

283

284

285

286

287

288

289

290

291

292

293

294

\section{Relative Velocity Data Distributions in Response to Physiological Stimuli}

We investigated the relative change distributions of velocity waveform characteristics from rest to hypercapnia and rest to exercise from the CFD and TCD datasets (Figure 3). We found that the relative change in velocity metrics from rest to hypercapnia were similar between CFD and TCD (all P>0.05), with the means of CFD relative velocity changes found to be slightly higher than the changes from TCD data for systolic ( $34 \pm 30 \%$ vs $28 \pm 12 \%, P=0.579$ ), average (44 $\pm 26 \%$ vs $39 \pm 15 \%, P=0.532)$ and end diastolic velocity $(58 \pm 33 \%$ vs $49 \pm 22 \%, P=0.477)$. A relative change in systolic velocity from a CFD case in response to hypercapnia was also observed to be greater than the higher quartile range limit. While small differences between CFD and TCD distributions were observed for average ( $22 \pm 27 \%$ vs $32 \pm 20 \%, P=0.323$ ) and end diastolic ( $19 \pm 29 \%$ vs $26 \pm 24 \%, P=0.566)$ velocities, the change in systolic velocity from rest to exercise was significantly higher in the TCD data compared to CFD $(40 \pm 18 \%$ vs $14 \pm 22 \%$, $\mathrm{P}=0.006)$. 


\section{Rest $\rightarrow$ Hypercapnia $\quad$ Rest $\rightarrow$ Exercise}
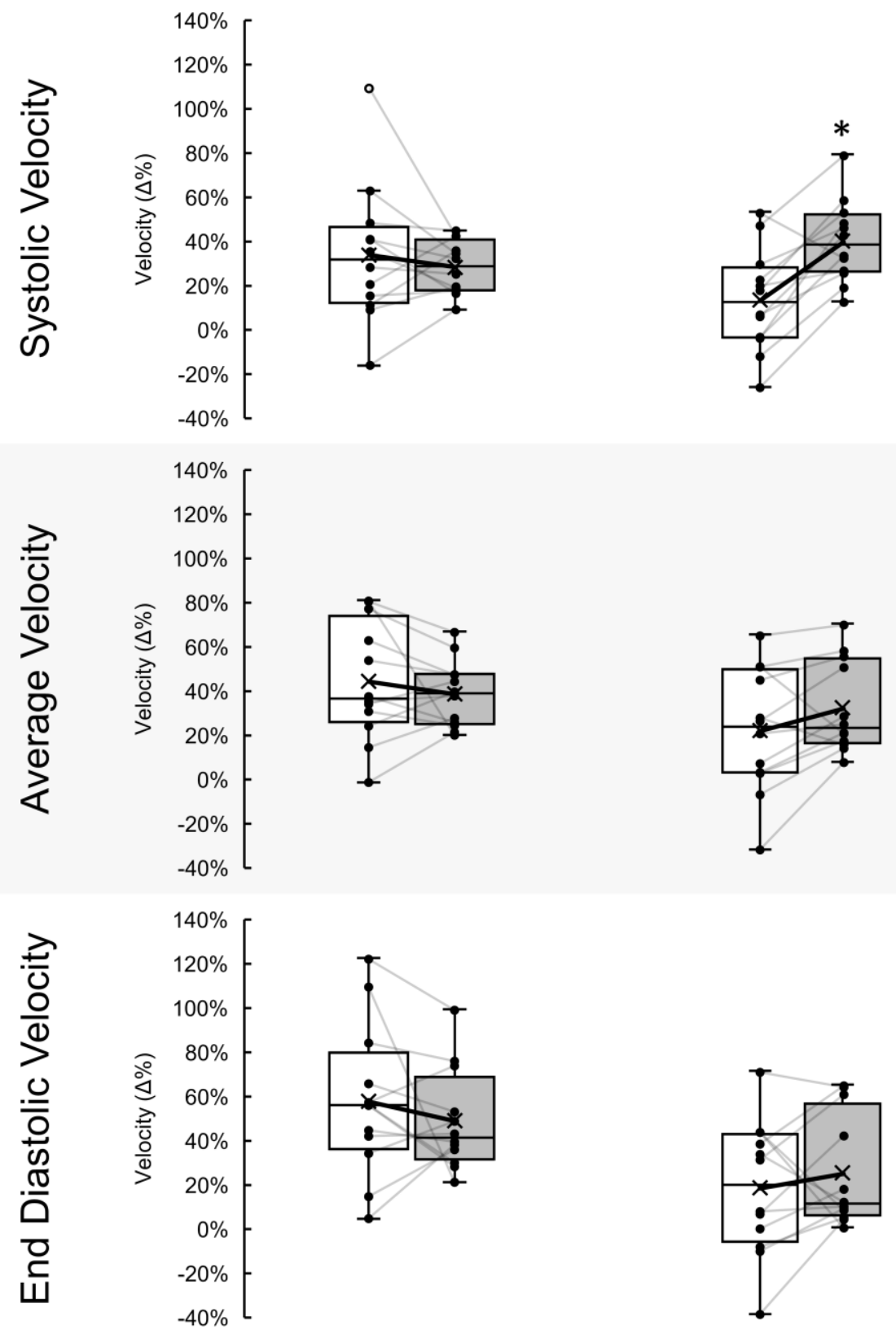

\section{$\square$ CFD Data $\square$ TCD Data}

296 Figure 3. Box plot distributions of relative change $(\Delta \%)$ in systolic, average and end diastolic maximal velocity extracted from CFD (white; $n=12 ; 6$ male, 6 female) and three cycle averaged TCD (grey; $n=12 ; 6$ male, 6 female) data between the conditions of rest to hypercapnia and rest to exercise in the right M1 segment. Individual differences between CFD and TCD relative 
change data are presented as black dots with grey connecting lines. The solid black line data point of relative change in systolic velocity from rest to hypercapnia that is outside the higher quartile range limit is displayed as a hollow circle. Stars $(*)$ indicate the level of significance $\left({ }^{*} \mathrm{P}<0.05 ;{ }^{*} \mathrm{P}<0.001\right)$ using t-tests between CFD and TCD data.

\section{Absolute Velocity Data Correlations at Rest and Physiological Stimuli}

307 For systolic, average and end diastolic velocity under the conditions of rest (Figure 4),

308 hypercapnia (Figure 5) and exercise (Figure 6), we found weak positive non-significant relationships (all $P>0.05$ ) that exhibited wide ranging limits of agreement and negative biases.

310 Correlations between absolute velocity characteristics from CFD and TCD sources with total average flow from the ICAs and VAs across all stimuli were also computed (see Supplementary Material, Figure S2). Correlations of total average inlet blood flow to velocity from the CFD data were all significantly correlated (all $\mathrm{P} \leq 0.05$ ), while TCD data yielded very mild non-

314 significant correlations (all $\mathrm{P}>0.05$ ), irrespective of exposure conditions or velocity metric. 


\section{Rest}
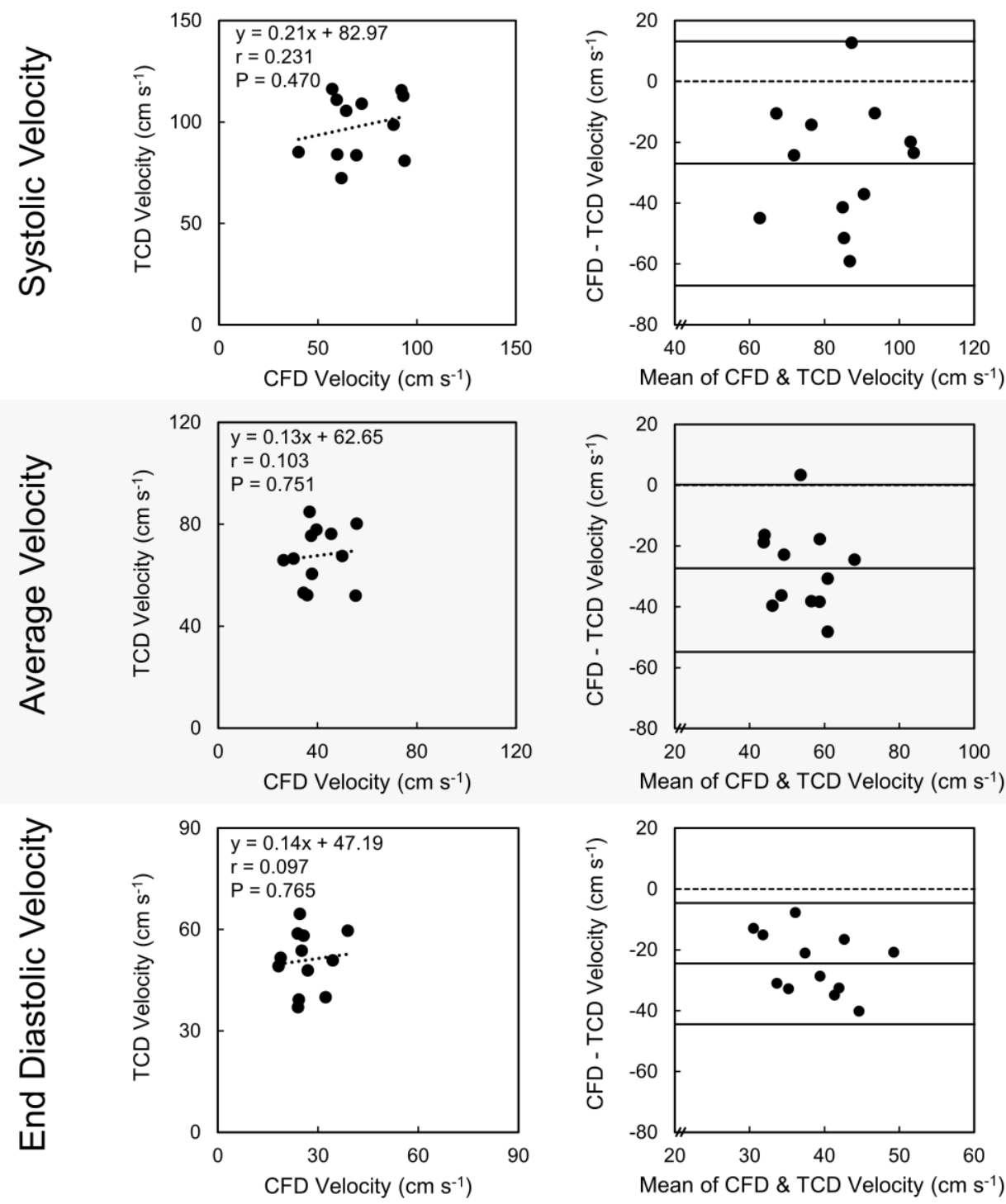

317 Figure 4. Correlation plots (left) and corresponding Bland-Altman plots (right) for absolute

318 systolic, average and end diastolic maximal velocity extracted from CFD (bottom axis; $n=12$;

3196 male, 6 female) and three cycle averaged TCD (left axis; n=12; 6 male, 6 female) data in the 320 right M1 segment. These data are collected from the rest condition. The linear regression

321 equation, Pearson's correlation coefficient $(r)$ and $p$-value $(P)$ are displayed for each 322 correlation plot. 
Hypercapnia
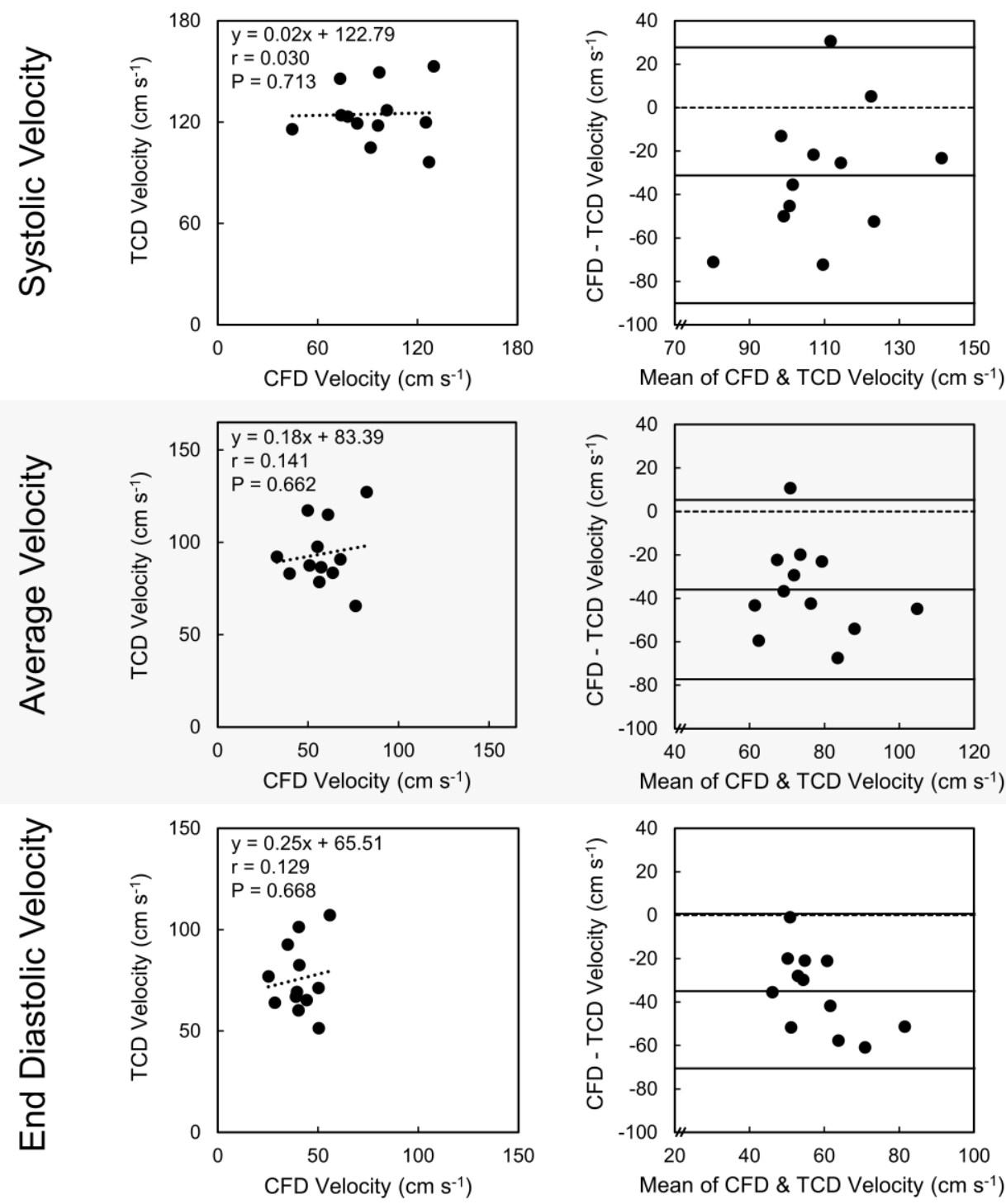

325 Figure 5. Correlation plots (left) and corresponding Bland-Altman plots (right) for absolute 326 systolic, average and end diastolic maximal velocity extracted from CFD (bottom axis; $n=12$;

3276 male, 6 female) and three cycle averaged TCD (left axis; n=12; 6 male, 6 female) data in the 328 right $\mathrm{M} 1$ segment. These data are collected from the hypercapnia condition. The linear regression equation, Pearson's correlation coefficient $(r)$ and $p$-value $(P)$ are displayed for each correlation plot. 


\section{Exercise}
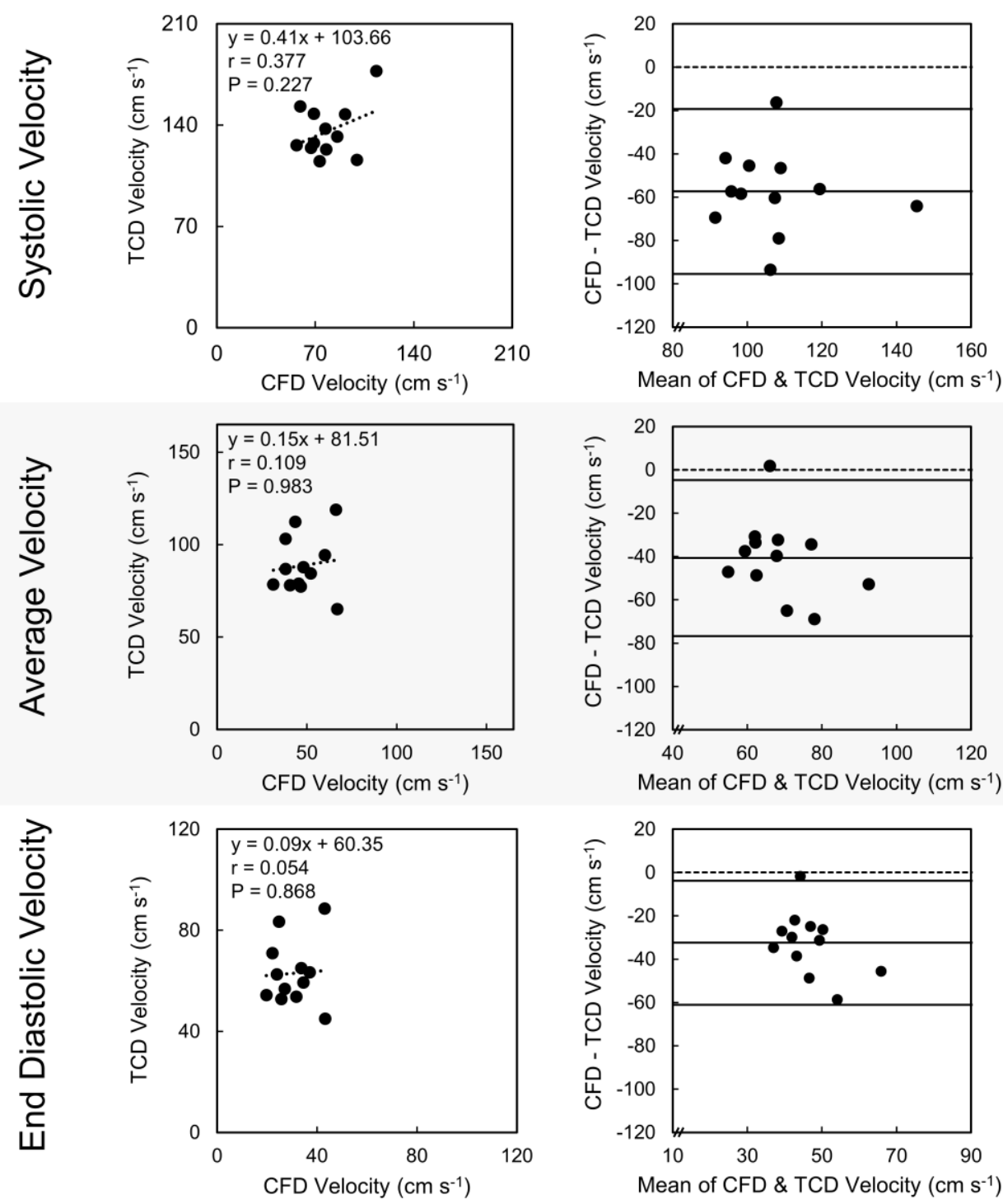

333 Figure 6. Correlation plots (left) and corresponding Bland-Altman plots (right) for absolute

334 systolic, average and end diastolic maximal velocity extracted from CFD (bottom axis; $n=12$;

3356 male, 6 female) and three cycle averaged TCD (left axis; n=12; 6 male, 6 female) data in the

336 right $\mathrm{M} 1$ segment. These data are collected from the exercise condition. The linear regression equation, Pearson's correlation coefficient $(r)$ and $p$-value $(P)$ are displayed for each correlation plot. 
Caddy et al. Comparison of TCD and CFD in response to stimuli

340

341 to exercise $(r=0.588, P=0.044)$, these correlations remained mild and non-significant.

\section{Relative Velocity Data Correlations in Response to Physiological Stimuli}

In comparison, correlations of CFD to TCD data for the relative change in velocity waveform characteristics of systolic, average and end diastolic velocity from rest to hypercapnia (Figure 7) and rest to exercise (Figure 8) yielded stronger moderate positive correlations, although limits of agreement remained large. Changes in systolic $(r=0.588, P=0.04)$ and average $(r=0.577, P=0.05)$ velocity from rest to hypercapnia were significantly correlated, with wide limits of agreement $(-46 \%$ and $57 \%,-38 \%$ and $49 \%)$, while end diastolic velocity $(r=0.448$, $P=0.14)$ was not significantly correlated and exhibited larger limits of agreement $(-54 \%$ and $72 \%)$. Similarly, for the change from rest to exercise, relative changes in systolic ( $r=0.604$, $P=0.04)$ and average $(r=0.770, P<0.01)$ velocity were found to be significantly correlated between CFD and TCD data, again with wide ranging limits of agreement (-64\% and $11 \%$, $46 \%$ and $25 \%)$, while end diastolic $(r=0.508, P=0.09)$ velocity was not significantly correlated and again displayed the largest range of limits of agreement (-62\% and $48 \%)$. Excluding relative systolic velocity change bias from rest to exercise $(-27 \%)$, the biases fell within $\pm 10 \%$ for all other velocity metrics and exposure conditions. A mild positive proportional bias was observed across most relative velocity change metrics. We also investigated correlations between relative changes in velocity characteristics from CFD and TCD sources with relative change in total average flow from the ICAs and VAs in response to stimuli (see Supplementary Material, Figure S3). We again observed strong positive and significant correlations between average total inlet blood flow and the relative change in MCA velocity calculated from CFD simulations across both responses to hypercapnia and exercise (all $\mathrm{P}<0.05$ ). Relative changes in TCD velocity metrics all remained positive, however, outside of average velocity from rest Nonetheless, the mild positive correlations for relative change data were stronger across all 

with inlet flow using absolute data.
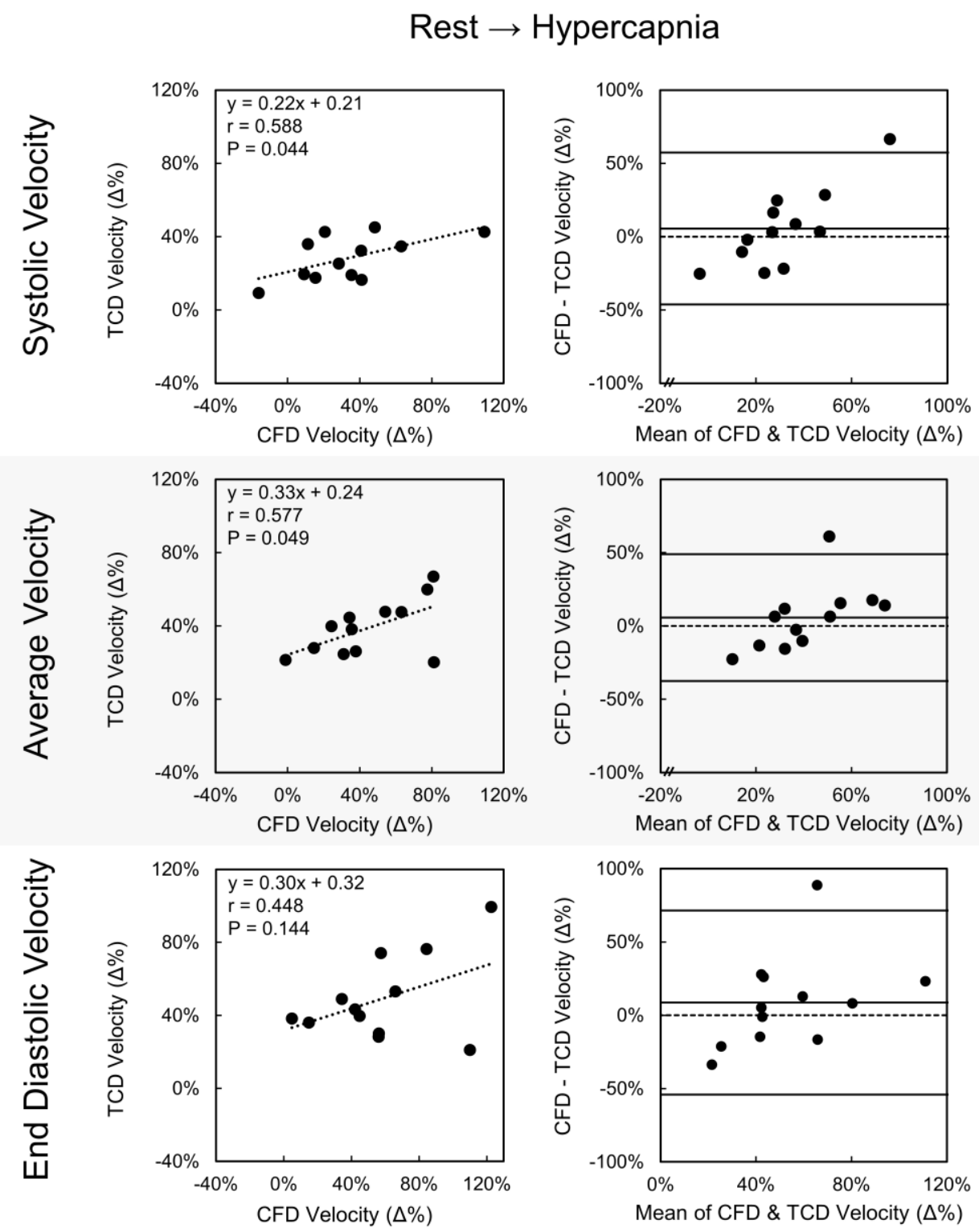

367 Figure 7. Correlation plots (left) and corresponding Bland-Altman plots (right) of the relative change $(\Delta \%)$ in systolic, average and end diastolic maximal velocity extracted from CFD

369 (bottom axis; $n=12 ; 6$ male, 6 female) and three cycle averaged TCD (left axis; $n=12 ; 6$ male, 


\section{Rest $\rightarrow$ Exercise}
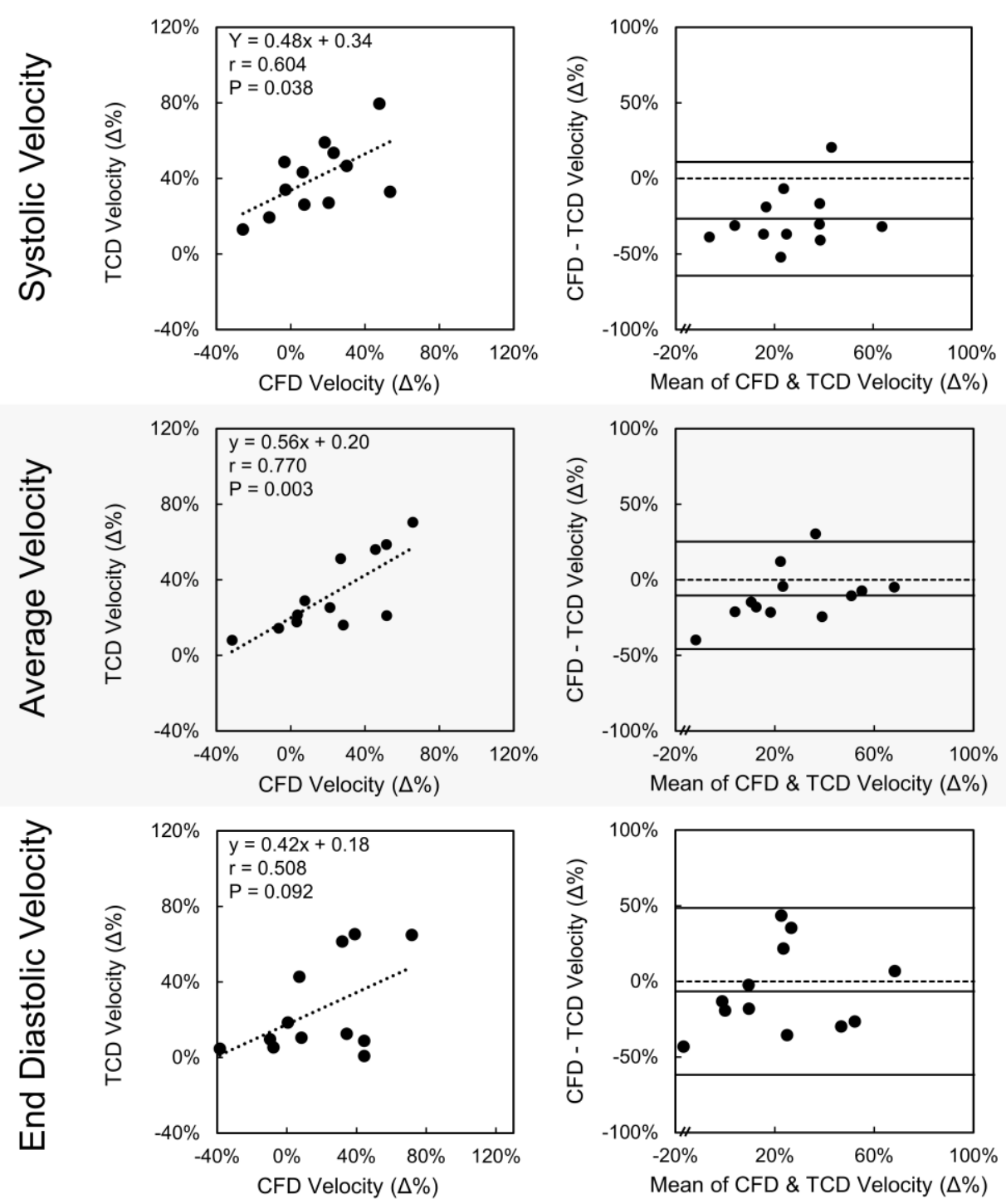

374 Figure 8. Correlation plots (left) and corresponding Bland-Altman plots (right) of the relative change $(\Delta \%)$ in systolic, average and end diastolic maximal velocity extracted from CFD

376 (bottom axis; $n=12 ; 6$ male, 6 female) and three cycle averaged TCD (left axis; $n=12 ; 6$ male, 
Caddy et al. Comparison of TCD and CFD in response to stimuli

379

380

381

382

383

384

385

386

387

388

389

390

391

392

393

394

395

396

397

398

399

400

401

402

\section{DISCUSSION}

In this study we investigated the differences in velocity obtained from two sources, subjectspecific CFD simulations and TCD ultrasound, under the three exposure conditions of rest, hypercapnia and exercise. In contrast to our initial hypothesis, we found that absolute TCD velocities were significantly higher than those calculated using CFD, and that these comparative data sets were uncorrelated, irrespective of exposure condition. This disparity between velocity data could be explained by underestimation from CFD simulations, overestimation from TCD methods, or a combination thereof. Despite the differences in absolute data observed between TCD and CFD methods, changes in response to each physiological condition were more similar with moderate to strong correlation.

Comparison of relative changes in velocity may minimize the impact of systematic sources of variability, since relative changes could serve to reduce error through cancellation. In concert with this mathematical explanation, it is also plausible that physiological variability may be reduced when a standardized stimulus (hypercapnia, exercise) is applied, compared to the multiple physiological mechanisms that combine to influence absolute data. It is also important to note, however, that although bias was reduced and correlations improved in the response to stimuli, there was still some evidence of proportional bias in the Bland-Altman plots, along with variable limits of agreement. These data indicate there may be range of improved agreement between either sources for moderate individual increase responses to stimuli, as opposed to low or high responses. This is also reflected in greater agreement for the more moderate responses from rest to exercise, compared to the larger changes found with rest to hypercapnia. While both of these physiological stimuli are associated with some degree of hypercapnia, $6 \% \mathrm{CO}_{2}$ exposure provides a much larger stimulus. Hypercapnia is a 
Caddy et al. Comparison of TCD and CFD in response to stimuli

403

404

405

406

407

408

409

410

411

412

413

414

415

416

417

418

419

420

421

422

423

424

425

426

powerful cerebral vasodilator, inducing possible MCA diameter change. The assumption of

fixed 3D geometry in CFD simulations may be associated with more error and variability in increased hypercapnic states, potentially explaining the higher correlations we observed in response to exercise than the $6 \% \mathrm{CO}_{2}$ exposure condition. In any event, reporting relative changes in velocity in response to stimuli may be an important adjunct to comparison of absolute measures in future studies.

One explanation for the disparity between CFD and TCD estimates of absolute velocity is that

CFD calculated velocities could be influenced by variability in the ultrasound derived input flows, collected in the ICAs and VAs. Although flow data derived from duplex ultrasound have been found to correlate with MR based estimates ${ }^{26}$, variability in this data can be high due difficulties associated with neck insonation and operator-related issues. Interestingly however, ultrasound measurements have been found to overestimate blood flow velocity compared to MR imaging (30), indicating that low CFD data in the current study may not be ascribed to low ultrasound derived flow inputs alone. Furthermore, average ICA and VA ultrasound flows in our study were similar to those observed in previous experiments $26,32,33$, 37. Whilst these findings suggest that the inputs to CFD were likely to be reliable, the combination of multiple data sources (ultrasound diameter and velocity, MRI-derived 3D geometry) along with CFD modelling assumptions may nonetheless have contributed to an underestimation of velocities using the CFD approach. Alternatively, an overestimation of velocity derived from the TCD approach may have occurred, due to phenomenon such as spectral broadening which can exaggerate peak blood flow velocity by up to $35 \%$ when lower megahertz probes are used ${ }^{12,16}$. The TCD and CFD data may also have differed due to the rigid wall modelling assumption used in CFD calculation, although the degree to which 
Caddy et al. Comparison of TCD and CFD in response to stimuli

427

428

429

430

431

432

433

434

435

436

437

438

440

441

442

443

444

445

446

447

changes in MCA diameter impact velocity remains a matter of debate. However, as an example, the incorporation of a dilation of the MCA for a given inlet flow would likely further reduce the velocity calculated from the CFD simulations and exacerbate the discrepancies found in this study. Finally, we investigated the association between variables using correlation between inlet flow to the brain (derived from duplex ultrasound) and the absolute (see Supplementary Material, Figure S2) and relative change (see Supplementary Material, Figure S3) in MCA velocity calculated using either CFD simulations or directly measured with TCD. We found that CFD calculated absolute and relative change in velocity were significantly correlated with the inlet flows, whereas TCD velocity was mostly uncorrelated with duplex ultrasound inlet data, irrespective of the exposure conditions. Consequently, the CFD simulations are likely to reflect, but also potentially exacerbate any changes in the calculated inlet flows from the neck arteries. These data provide insight regarding the capacity of CFD and TCD to accurately reflect absolute brain blood flow in humans.

Although predicated on different hypotheses, two previous papers have compared TCD data with CFD simulations. Jahed et al. compared velocity measurements from two cerebral aneurysmal cases with corresponding TCD data in the anterior, middle and posterior cerebral arteries ${ }^{17}$. Differences in velocity measurements were observed in the MCA between CFD and TCD sources and the authors concluded that TCD tests may introduce error and possibly lead to incorrect decisions regarding clinical diagnosis and treatment. CFD methods have also been used in the context of investigating sickle cell anemia ${ }^{30}$. The findings indicated that misplacement of the TCD sampling and averaging region within a localized region of low or high velocity in the MCA could also lead to misdiagnosis. Our findings in 12 healthy subjects add to these previous experiments, in showing that TCD and CFD approaches to velocity 
assessment can differ, at least when absolute comparisons between derived velocities are compared.

453

454 Although validation of either dataset is difficult to ascertain, the question of whether TCD or

455 CFD approaches provide a more accurate reflection of the true changes in velocity from physiological stimulation can be somewhat informed by consideration of previous studies which have compared techniques to magnetic resonance approaches. Seitz et al. found that TCD velocities exceeded MRA measured velocities by around $30 \%$ and reported low correlations between these approaches ${ }^{34}$. Chang et al. also reported $30 \%$ greater velocities via $\operatorname{TCD}^{8}$, but found that phase contrast MRA techniques correlated strongly with TCD. $A$ study by Meckel et al. compared 4D phase contrast MRI (4D PCMRI) and transcranial colorcoded duplex sonography ${ }^{23}$ and also found TCD derived data was higher than 4D PCMRI, along with weak to mild correlations between these approaches. Leung et al. reported higher peak velocities using TCD than PCMRA ${ }^{21}$ but also reported strong correlations when resting and hypercapnia-derived data were compared between approaches. Taken together, these studies indicate that, at rest and in response to physiological stimuli, TCD approaches may present higher velocities when compared to MR based methods, but the degree to which they correlate is variable. However, despite the variabilities between in sources, our results suggest that either technique (TCD or CFD) remain practical and beneficial in understanding the relative change in velocities in response to physiological stimuli in the brain.

472 Although we believe the methods used in this study to investigate comparisons between CFD and TCD data are rigorous, and note that they have been previously published ${ }^{41}$, the present 474 study is not without its limitations. Our cohort was limited to young, healthy individuals with 
475

476

477

478

480

481

482

no pre-existing cardiovascular diseases. Consequently, our findings may vary from some aforementioned studies due to their use of aging cohorts, or individuals with existing cardiovascular diseases or risk factors. The MRA scans that were segmented to produce the 3D models used in our study were performed with resting supine participants. Ideally, provided availability of specialized equipment, MRA scans should be captured in response to exposure conditions, allowing any changes in vessel diameter to be embedded in future CFD simulations. Ultrasound imaging was performed in a recumbent position during exposure conditions while a resting supine body position was required for the MRA scans. Although matching of imaging positions is preferable, performing ultrasound imaging outside of a recumbent position is difficult during exercise ${ }^{38}$. Although care was made to ensure placement of sampling regions was consistent between TCD measurement and CFD simulations, this sampling was still operator dependent. While we used previously established methods ${ }^{20,41}$ for ultrasound waveform averaging, additional averaging of cardiac cycles may also serve to reduce the variability in results. In the CFD simulations, outlet boundary conditions were distributed using resting regional flow measurements derived from literature and diameter-based flow splitting exponents which were constant across all outlets. In the absence of regional brain blood flow data, particularly in response to stimuli, an exponent value appropriate for cerebrovascular vessels was used ${ }^{41}$, however research has suggested that this exponent may vary for each individual outlet ${ }^{9,10}$. A localized outlet splitting method as described by Chnafa et al. ${ }^{10}$, provided access to flow data in the brain, may be more appropriate in future CFD based cerebrovascular research. Our simulations employed rigid wall modelling as the resolution of the MRA data collected was unable to resolve arterial wall thickness and subject specific material properties were not known. Alternatively, implementation of fluid structure interaction (FSI) modelling would allow the vessel wall to 
deform and absorb energy throughout the cardiac cycle. However, this would likely reduce

500 the velocities calculated from CFD simulation - further exacerbating the discrepancies

501 observed between TCD and CFD velocities. True validation of CFD methods was unable to be

502 performed in this study due to imaging limitations. Independently captured time varying

503 image datasets using 4D MRI methods may help provide validation of future cerebrovascular

504 CFD simulations. Finally, with only 12 participants, the number of cases investigated in this

505 study is relatively small. However, despite the low number of cases, we still observed

506 statistically significant results which may have important implications for future physiological

507 research.

508

509 In conclusion, we aimed to compare velocity measurements obtained within the MCA under

510 resting and external physiological stimuli (hypercapnia, exercise) conditions using TCD

511 ultrasound and independently constructed CFD simulations. Although we found discrepancies

512 between absolute velocity data obtained between CFD and TCD approaches, measurements

513 of relative velocity characteristics in response to different stimuli from rest showed improved

514 but variable agreement, with the strongest correlations observed for the change in average

515 velocity between rest and exercise. Therefore, in addition to absolute measurements,

516 incorporation of relative changes in velocity in response to physiological stimuli is an

517 important consideration for future research using either TCD ultrasound or CFD

518 cerebrovasculature simulations. 
Caddy et al. Comparison of TCD and CFD in response to stimuli

\section{ACKNOWLEDGEMENTS}

520 We acknowledge the resources provided by the Pawsey Supercomputing Centre with funding

521 from the Australian Government and the Government of Western Australia. D.J.G. is

522 supported by a NHMRC Principal Research Fellowship (APP1080914). There are no conflicts

523 of interest.

524

\section{Author Contributions}

526 H.T.C., H.J.T., D.J.G., and B.J.D. conceived and designed research; H.J.T., K.J.S. and D.J.G.

527 performed experiments; H.T.C., L.J.K. and B.J.D. developed simulations; H.T.C., H.J.T., L.J.K., 528 K.J.S., D.J.G., and B.J.D. interpreted results of experiments; H.T.C, B.J.D. and D.J.G. drafted 529 manuscript; H.T.C., H.J.T., L.J.K., K.J.S., B.J.D. and D.J.G. edited and revised manuscript; H.T.C., 530 H.J.T., L.J.K., K.J.S., B.J.D. and D.J.G. approved final version of manuscript; H.T.C., L.J.K., and 531 B.J.D. analyzed data; H.T.C., L.J.K., B.J.D. and D.J.G. prepared figures. 
Caddy et al. Comparison of TCD and CFD in response to stimuli

532

533

534

535

536

537

538

539

540

541

542

543

544

545

546

547

548

549

550

551

552

553

554

555

556

557

\section{REFERENCES}

1. Ainslie P. N. and R. L. Hoiland. Transcranial Doppler ultrasound: Valid, invalid, or both? Journal of Applied Physiology 117: 1081-1083, 2014.

2. Alnæs M. S., J. Isaksen, K.-A. Mardal, B. Romner, M. K. Morgan and T. Ingebrigtsen. Computation of Hemodynamics in the Circle of Willis. Stroke 38: 2500-2505, 2007.

3. Armstead W. M. Cerebral Blood Flow Autoregulation and Dysautoregulation. Anesthesiology clinics 34: 465-477, 2016.

4. Barlinn K., S. Kolieskova, R. Bavarsad Shahripour, J. Kepplinger, A. K. Boehme, T. Siepmann, V. Puetz, U. Bodechtel, W. D. Jordan and A. V. Alexandrov. Increased Pulsatility of the Intracranial Blood Flow Spectral Waveform on Transcranial Doppler Does Not Point to Peripheral Arterial Disease in Stroke Patients. Journal of Stroke and Cerebrovascular Diseases 24: 189-195, 2015.

5. Bishop C. C., S. Powell, D. Rutt and N. L. Browse. Transcranial Doppler measurement of middle cerebral artery blood flow velocity: a validation study. Stroke 17: 913-915, 1986.

6. Bluestein D., L. Niu, R. T. Schoephoerster and M. K. Dewanjee. Fluid mechanics of arterial stenosis: Relationship to the development of mural thrombus. Annals of Biomedical Engineering 25: 344, 1997.

7. Brothers R. M. and R. Zhang. CrossTalk opposing view: The middle cerebral artery diameter does not change during alterations in arterial blood gases and blood pressure. The Journal of physiology 594: 4077-4079, 2016.

8. Chang W., B. Landgraf, K. M. Johnson, S. Kecskemeti, Y. Wu, J. Velikina, H. Rowley, O. Wieben, C. Mistretta and P. Turski. Velocity Measurements in the Middle Cerebral Arteries of Healthy Volunteers Using 3D Radial Phase-Contrast HYPRFlow: Comparison with Transcranial Doppler Sonography and 2D Phase-Contrast MR Imaging. American Journal of Neuroradiology 32: 54-59, 2011. 9. Chnafa C., P. Bouillot, O. Brina, B. M. A. Delattre, M. I. Vargas, K. O. Lovblad, V. M. Pereira and D. A. Steinman. Vessel calibre and flow splitting relationships at the internal carotid artery terminal bifurcation. Physiol Meas 38: 2044-2057, 2017. 
Caddy et al. Comparison of TCD and CFD in response to stimuli

558 10. Chnafa C., O. Brina, V. M. Pereira and D. A. Steinman. Better Than Nothing: A Rational

559 Approach for Minimizing the Impact of Outflow Strategy on Cerebrovascular Simulations. AJNR.

560 American journal of neuroradiology 39: 337-343, 2018.

561 11. Coverdale N. S., J. S. Gati, O. Opalevych, A. Perrotta and J. K. Shoemaker. Cerebral blood flow

562 velocity underestimates cerebral blood flow during modest hypercapnia and hypocapnia. Journal of

563 Applied Physiology 117: 1090-1096, 2014.

564 12. Eicke B. M., F. W. Kremkau, H. Hinson and C. H. Tegeler. Peak velocity overestimation and

565 linear-array spectral Doppler. J Neuroimaging 5: 115-121, 1995.

566 13. Ghorbani A., M. J. Ahmadi and H. Shemshaki. The value of transcranial Doppler derived

567 pulsatility index for diagnosing cerebral small-vessel disease. Adv Biomed Res 4: 54, 2015.

568 14. Groen D., R. A. Richardson, R. Coy, U. D. Schiller, H. Chandrashekar, F. Robertson and P. V. 569 Coveney. Validation of Patient-Specific Cerebral Blood Flow Simulation Using Transcranial Doppler 570 Measurements. Front Physiol 9: 721, 2018.

571 15. Hoiland R. L., K. J. Smith, H. H. Carter, N. C. S. Lewis, M. M. Tymko, K. W. Wildfong, A. R. Bain,

572 D. J. Green and P. N. Ainslie. Shear-mediated dilation of the internal carotid artery occurs independent

573 of hypercapnia. American Journal of Physiology-Heart and Circulatory Physiology 313: H24-H31, 2017.

574 16. Hoskins P. R. Accuracy of maximum velocity estimates made using Doppler ultrasound systems. Br J Radiol 69: 172-177, 1996.

576 17. Jahed M., F. Ghalichi and M. Farhoudi. Comparison of blood velocity between Transcranial

577 Doppler and numerical method in the patient-specific Circle of Willis with aneurysm. Biomed Mater

578 Eng 30: 427-438, 2019.

579 18. Kidwell C. S., S. el-Saden, Z. Livshits, N. A. Martin, T. C. Glenn and J. L. Saver. Transcranial 580 Doppler pulsatility indices as a measure of diffuse small-vessel disease. J Neuroimaging 11: 229-235, 5812001.

582 19. Kim C. S., C. Kiris, D. Kwak and T. David. Numerical simulation of local blood flow in the carotid and cerebral arteries under altered gravity. J Biomech Eng 128: 194-202, 2006. 
584 20. Kurmanavichius J., H. Baumann, R. Huch and A. Huch. Determination of the minimum number 585 of cardiac cycles necessary to ensure representative blood flow velocity measurements. J Perinat Med 17: 33-39, 1989.

587

21. Leung J., A. Behpour, N. Sokol, A. Mohanta and A. Kassner. Assessment of intracranial blood flow velocities using a computer controlled vasoactive stimulus: A comparison between phase contrast magnetic resonance angiography and transcranial doppler ultrasonography. Journal of Magnetic Resonance Imaging 38: 733-738, 2013.

591 22. Levitt M. R., M. C. Barbour, S. Rolland du Roscoat, C. Geindreau, V. K. Chivukula, P. M. McGah, coiling using high-resolution and high-energy synchrotron X-ray microtomography: comparison with the homogeneous porous medium approach. J Neurointerv Surg 9: 0-0, 2017. transcranial ultrasound techniques and 2D PC MRI. Neuroradiology 55: 389-398, 2013.

602 25. Naqvi J., K. H. Yap, G. Ahmad and J. Ghosh. Transcranial Doppler Ultrasound: A Review of the

603 Physical Principles and Major Applications in Critical Care. International Journal of Vascular Medicine 604 2013: 629378, 2013.

605 26. Oktar S. O., C. Yücel, D. Karaosmanoglu, K. Akkan, H. Ozdemir, N. Tokgoz and T. Tali. Blood606 Flow Volume Quantification in Internal Carotid and Vertebral Arteries: Comparison of 3 Different 607 Ultrasound Techniques with Phase-Contrast MR Imaging. American Journal of Neuroradiology 27: 363608 369, 2006. 
Caddy et al. Comparison of TCD and CFD in response to stimuli

27. Panerai R. B., J. L. Jara, N. P. Saeed, M. A. Horsfield and T. G. Robinson. Dynamic cerebral autoregulation following acute ischaemic stroke: Comparison of transcranial Doppler and magnetic resonance imaging techniques. Journal of Cerebral Blood Flow \& Metabolism 36: 2194-2202, 2016.

28. Phillips A. A., F. H. Chan, M. M. Zheng, A. V. Krassioukov and P. N. Ainslie. Neurovascular coupling in humans: Physiology, methodological advances and clinical implications. J Cereb Blood Flow Metab 36: 647-664, 2016.

29. Purkayastha S. and F. Sorond. Transcranial Doppler ultrasound: technique and application. Seminars in neurology 32: 411-420, 2012.

30. Rivera C. P., A. Veneziani, R. E. Ware and M. O. Platt. Original Research: Sickle cell anemia and pediatric strokes: Computational fluid dynamics analysis in the middle cerebral artery. Experimental Biology and Medicine 241: 755-765, 2016.

31. Roache P. J. Perspective: A Method for Uniform Reporting of Grid Refinement Studies. Journal of Fluids Engineering 116: 405-413, 1994.

32. Sato K., J. P. Fisher, T. Seifert, M. Overgaard, N. H. Secher and S. Ogoh. Blood flow in internal carotid and vertebral arteries during orthostatic stress. Experimental physiology 97: 1272-1280, 2012. 33. Sato K. and T. Sadamoto. Different blood flow responses to dynamic exercise between internal carotid and vertebral arteries in women. Journal of Applied Physiology 109: 864-869, 2010.

34. Seitz J., M. Strotzer, J. Schlaier, W. R. Nitz, M. Völk and S. Feuerbach. Comparison between magnetic resonance phase contrast imaging and transcranial Doppler ultrasound with regard to blood flow velocity in intracranial arteries: work in progress. J Neuroimaging 11: 121-128, 2001.

35. Shen Y., Y. Wei, R. P. H. Bokkers, M. Uyttenboogaart and J. M. C. van Dijk. Study protocol of validating a numerical model to assess the blood flow in the circle of Willis. BMJ Open 10: e036404, 2020.

36. Shojima M., M. Oshima, K. Takagi, R. Torii, M. Hayakawa, K. Katada, A. Morita and T. Kirino. Magnitude and role of wall shear stress on cerebral aneurysm: computational fluid dynamic study of 20 middle cerebral artery aneurysms. Stroke 35: 2500-2505, 2004. 
635

636

637

638

639

640

641

642

643

644

645

646

647

648

649

650

651

652

653

654

655

656

657

37. Skytioti M., S. Søvik and M. Elstad. Internal carotid artery blood flow in healthy awake subjects is reduced by simulated hypovolemia and noninvasive mechanical ventilation. Physiological Reports 4: e12969, 2016.

38. Smith K. J. and P. N. Ainslie. Regulation of cerebral blood flow and metabolism during exercise. Experimental physiology 102: 1356-1371, 2017.

39. Smith K. J., R. L. Hoiland, R. Grove, H. McKirdy, L. Naylor, P. N. Ainslie and D. J. Green. Matched increases in cerebral artery shear stress, irrespective of stimulus, induce similar changes in extracranial arterial diameter in humans. J Cereb Blood Flow Metab 39: 849-858, 2019.

40. Smith K. J., K. W. Wildfong, R. L. Hoiland, M. Harper, N. C. Lewis, A. Pool, S. L. Smith, T. Kuca, G. E. Foster and P. N. Ainslie. Role of $\mathrm{CO} 2$ in the cerebral hyperemic response to incremental normoxic and hyperoxic exercise. Journal of applied physiology (Bethesda, Md. : 1985) 120: 843-854, 2016.

41. Thomas H. J., U. Rana, C. E. Marsh, H. T. Caddy, L. J. Kelsey, K. J. Smith, D. J. Green and B. J. Doyle. Assessment of cerebrovascular responses to physiological stimuli in identical twins using multimodal imaging and computational fluid dynamics. Journal of Applied Physiology 129: 1024-1032, 2020.

42. Willie C. K., F. L. Colino, D. M. Bailey, Y. C. Tzeng, G. Binsted, L. W. Jones, M. J. Haykowsky, J. Bellapart, S. Ogoh, K. J. Smith, J. D. Smirl, T. A. Day, S. J. Lucas, L. K. Eller and P. N. Ainslie. Utility of transcranial Doppler ultrasound for the integrative assessment of cerebrovascular function. J Neurosci Methods 196: 221-237, 2011.

43. Willie C. K., D. B. Macleod, A. D. Shaw, K. J. Smith, Y. C. Tzeng, N. D. Eves, K. Ikeda, J. Graham, N. C. Lewis, T. A. Day and P. N. Ainslie. Regional brain blood flow in man during acute changes in arterial blood gases. The Journal of physiology 590: 3261-3275, 2012. 